\title{
Computation of dust charge and potential on a static spherical dust grain immersed in rf discharges
}

\author{
V.R. Ikkurthi* ${ }^{1}$ K. Matyash ${ }^{2}$ A. Melzer, ${ }^{1}$ and R. Schneider ${ }^{2}$ \\ ${ }^{1}$ Ernst-Moritz-Arndt Universitat Greifswald, 17489 Greifswald, Germany. \\ ${ }^{2}$ Max-Planck Institut fur Plasmaphysik, \\ EURATOM Association, 17491 Greifswald, Germany.
}

(Dated: March 10, 2009)

\begin{abstract}
Dust charge and potential on static spherical dust grains located in an argon rf discharge under typical laboratory experiment conditions have been computed using a three-dimensional ParticleParticle-Particle-Mesh $\left(P^{3} M\right)$ code. Elastic and inelastic collisions have been included in the current model to obtain realistic rf discharge plasma conditions. Dust charge, potential and potential distribution around the dust have been computed for various sizes of dust placed at different locations in the rf discharge. The dust charge is found to be smaller than the values from simple Orbit Motion Limited (OML) model due to ion-neutral collisions. Further, the dust potential has been found to be increasing with dust-size. Moreover, the shielding length of the dust has been found between electron and ion Debye lengths.
\end{abstract}

\footnotetext{
* contact author: ramana@physik.uni-greifswald.de
} 


\section{INTRODUCTION}

Dusty (complex) plasmas are plasmas containing charged micron-sized particles. The dust grain attains a charge (positive or negative) due to various charging mechanisms ${ }^{1}$, such as absorption of electrons, ions, secondary electron emission, etc. Charging of a dust grain is determined by the background plasma properties, where collisions between various plasma species play an important role. The dust charge governs the electrostatic potential around the dust and interdust grain interaction and hence the formation of dust structures. Also the ion drag force - the force exerted on dust grain by traversing ions around the dust due to coulomb interaction - which plays a crucial role in phenomena such as wake-field formation in $\mathrm{rf}$ sheaths ${ }^{2}$ and dust void formation in microgravity dusty plasma experiments ${ }^{3}$, depends on the dust potential and dust charge. So, in order to quantify these various properties for realistic discharge conditions in rf discharges, dust charge and potential have to be determined accurately. Since in rf discharges the plasma bulk and sheath have different properties (e.g. streaming of ions), the dust charge and potential might vary for dust grains located at different positions in rf discharge.

In this work, three-dimensional kinetic simulations have been performed to quantify the dust charge and potential on spherical dust grains located at different positions in rf discharges. The computation of dust charges dates back to the earliest probe theories in the $1920 \mathrm{~s}^{4}$. Probe theories calculate the current to an electrostatic probe as a function of probe potential and probe shape. The floating potential is derived as the point where ion and electron currents balance. Later, probe theory has been applied to dust charging. First probe theories based on orbital motion, Orbit Motion Limited (OML) theory, was put forth by Mott-Smith and Langmuir ${ }^{4,5}$. Here, the angular momentum of the ions imposes a limit on the maximum ion current. OML does not include the existence of an absorption radius around dust and predicts a dust potential independent of dust size. There have been many refinements to the theory. Limitations and applicability of these theories have been dealt with previously in many articles ${ }^{5-15}$. More general Orbital Motion (OM) theory ${ }^{7,8}$ involves solving simultaneously for the surface potential, the potential distribution around the probe, and the distribution of ion trajectories. Radial Motion theory proposed by Allen, Boyd and Reynolds $(\mathrm{ABR})^{6}$ includes a pre-sheath transition region where quasi-neutrality is satisfied but potentials on the order of the Bohm potential ${ }^{13}$ are allowed to exist. 
In both $\mathrm{OM}$ and $\mathrm{ABR}$ theories, originally collisional effects between charged plasma species and the background gas have not been considered. It has been $\operatorname{shown}^{7}$ that due to collisions near the probe, some of the charged plasma particles can be trapped in the potential well and this could play a very important role, e.g. affect shielding lengths. Lampe et. al. ${ }^{14,15}$, Ratynskaia et. al. ${ }^{16}$ and Khrapak et. al. ${ }^{17}$ considered charge-exchange collisions between ions and neutrals, as these collisions are particularly effective in creating trapped ions. The above theories do not account for streaming ions leading to difficulties in determining the charge of dust located in the sheath. (However for $\mathrm{OML}^{5}$, streaming effects can be included in ion flux calculations.) Maxwellian distributions for the electrons are assumed in nearly all cases, and a variety of distributions from monoenergetic to Maxwellian are typically assumed for the ion distributions ${ }^{18-21}$. For rf plasmas, electrons have non-Maxwellian and sometimes bi-Maxwellian energy distributions ${ }^{22,23}$. Ions are also far from Maxwellian, as they become accelerated in sheaths acquiring a high streaming velocity.

For the sake of completeness, we mention different experimental techniques employed to derive the dust charge from experiments. These include oscillations in the sheath ${ }^{24-29}$, collisions of dust particle pairs ${ }^{30-33}$, waves $^{34,35}$, direct measurement with Faraday-cup ${ }^{36,37}$.

Kinetic simulations are promising tools for computations of dust charge, potential and potential distribution around dust for realistic experimental conditions. The reason is that these models include nearly all types of possible collisions, they track the trajectories of the plasma species and also account for non-Maxwellian distributions of the plasma species. Hutchinson et. al. ${ }^{38-42}$ performed kinetic simulations to compute ion currents to a probe. Zobnin et. al. ${ }^{43,44}$ performed kinetic simulations for an intermediate collisionality regime. Matyash et. al. ${ }^{45,46}$ performed kinetic simulation using a Particle-Particle-Particle-Mesh $\left(P^{3} M\right) \operatorname{code}^{45}$, to compute dust charge and potential on dust grains located in the bulk of rf discharges and in magnetized edge plasmas. Here, we extend the latter study to obtain more insight into collisional effects on the dust charging and shielding in rf discharges. In this work, we present the dust parameters computed for a single static spherical dust grain located at different positions in an rf discharge. We compare the simulation results with existing charging models. The dust potential distribution is compared with the Debye-Hückel potential, as it is widely used in the dusty plasma community. 


\section{II. $P^{3} M$ DESCRIPTION}

A detailed description of the $P^{3} M$ code used in our simulations can be found in $[45,47]$. In our plasma model, plasma particles (electrons and ions) are treated kinetically (Particlein-Cell with Monte Carlo Collisions (PIC-MCC)), which allows to self-consistently resolve the electrostatic sheath in front of a wall. A drawback of the PIC method is that the space resolution is limited by the size of the grid which is typically of the order of the Debye length (fraction of a millimeter for low temperature plasmas). The size of the dust grains is typically in the micrometer range and thus much smaller than the grid size. In conventional PIC algorithms, the plasma particles are represented by charged clouds of the grid size and are able to penetrate each other ${ }^{48}$. This leads to high inaccuracies for interparticle interaction when the distance becomes smaller than the cell size. In this case, the interaction force strongly deviates from the Coulomb force for small distances and tends to go to zero as the inter-particle distance decreases. Therefore, conventional PIC models are able to resolve long range (larger than the Debye length) interaction between the particles, but fail to resolve the close-range interaction for distances comparable to the radius of the dust grains. One approach to overcome this is to use a PIC-MC particle collision operator ${ }^{49}$ for describing plasma species absorption at the dust with the collision cross-section obtained from analytic $\mathrm{OML}^{5}$ theory. Another approach is to resolve or follow ion trajectories around dust until they are absorbed or scattered ${ }^{39,40,45,50}$. The present simulations are based on the latter approach.

In order to accurately resolve close-range interactions between dust grains and plasma particles, we extended our PIC model, combining it with a molecular dynamic (MD) algorithm. In the resulting Particle-Particle Particle-Mesh $\left(P^{3} M\right)$ model, the long-range interaction of the dust grains with charged particles of the background plasma is treated according to the PIC formalism. For particles which are closer to the dust grain than a Debye length their interaction force is computed according to a direct particle-particle MD scheme using the exact electrostatic potential. This is implemented in the following way: in the computational domain, the cell in which the dust grain is located together with the neighboring cells form the "MD" region. All particles outside the MD region are treated according to the conventional PIC scheme. For plasma particles (electrons and ions) inside the MD region the electric field is calculated as: $\mathrm{E}=E_{\text {grid }}+E_{\text {dust }}$. For the calculation of the grid field 
$E_{\text {grid }}$, we use the charge density as in the PIC part from which the dust grain contribution is subtracted. The dust contribution is accounted for by the exact Coulomb electric field $E_{\text {dust }}$ pairwise between the dust and the plasma particle. In order to resolve particle motion on scales of the order of the dust grain size, particles in the MD region are moved with a time step smaller than in the PIC region. Plasma particles which cross the computational dust grain boundary are assumed to be absorbed. The dust grain charge is updated each MD time step.

Collisions among electrons, various ion and neutral species govern the behavior of rf discharges. Ionization collisions between electrons and neutral species help in sustaining the plasma. Ion-neutral collisions in the bulk or the sheath determine the ion energy distributions (IEDs) and ion angular distributions (IADs). Hence, it is crucial to implement the various collisions between different species of the system to accurately simulate the system.

In the present $P^{3} M$ code, a binary Coulomb collision model described in $[47,51]$ has been implemented. Coulomb collisions between charged species, electron-impact ionization, efficient excitation, electron-argon elastic collisions and momentum transfer charge-exchange collisions were taken into account in the simulation. The collisions implemented for current study are listed in Table I.

TABLE I: List of collisions included in the current simulations

\begin{tabular}{lc}
\hline \hline Collision & Reference \\
\hline e-e, e-ion, ion-ion Coulomb & 52 \\
e-Ar Elastic Collision & 53 \\
$\mathrm{Ar}^{+}$- Ar Elastic Collisions & 53 \\
Ionization : e $+\mathrm{Ar} \rightarrow \mathrm{Ar}^{+}+2 \mathrm{e}$ & 53 \\
Charge-exchange $: \mathrm{Ar}+\mathrm{Ar}^{+} \rightarrow \mathrm{Ar}^{+}+\mathrm{Ar}$ & 53 \\
Excitation : e $+\mathrm{Ar} \rightarrow \mathrm{Ar}^{*}+\mathrm{e}$ & 53 \\
\hline \hline
\end{tabular}

\section{METHOD OF COMPUTATION}

We have applied the $P^{3} M$ model to investigate the dust grain charging process in a capacitive rf discharge in argon. The parameters of the simulation were chosen to represent 
the conditions of the experiments with Yukawa balls (see Ref. [54]). As background gas, argon with pressure $\mathrm{p}=50 \mathrm{~Pa}$ and neutral gas temperature $\mathrm{T}=300 \mathrm{~K}$ was used.

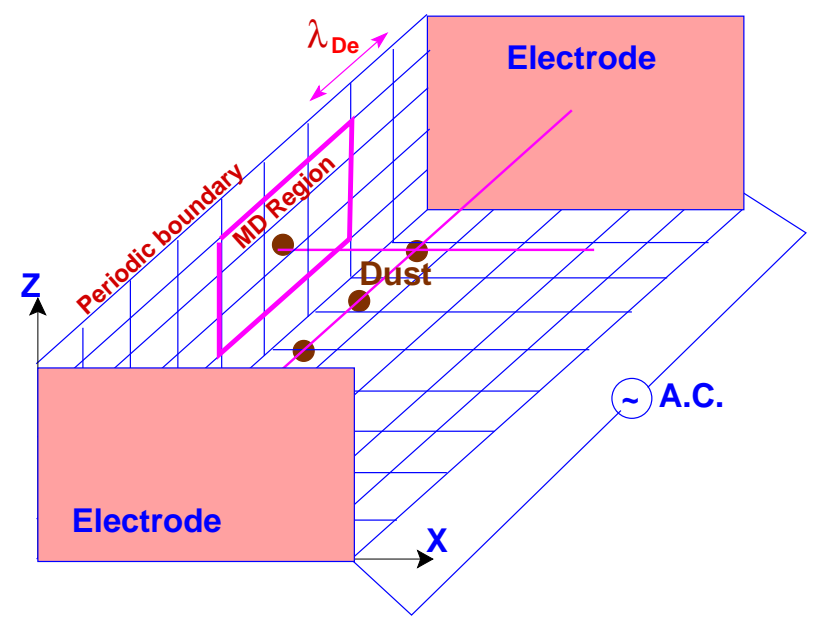

FIG. 1: (Color online) Schematic of 3D Computational Grid for simulation of Dust in rf discharges. The MD region (cube) is projected onto the left wall.

The computational domain representing the rf discharges and dust locations is shown in Fig. 1. The discharge system dimensions are taken as $0.15 \mathrm{~cm} \times 2.4 \mathrm{~cm} \times 0.15 \mathrm{~cm}$. The plasma volume is divided into $8 \times 128 \times 8$ computational cells (see Ref. [45] for selection criteria for cell-widths and time-step). The electrodes are aligned in the XZ-plane and the electrode separation is $2.4 \mathrm{~cm}$. The lower electrode at $\mathrm{Y}=0 \mathrm{~cm}$ is grounded and the upper electrode at $\mathrm{Y}=2.4 \mathrm{~cm}$ is powered with a sinusoidal voltage at frequency $\mathrm{f}_{r f}=13.56 \mathrm{MHz}$ and amplitude $U_{r f}=30 \mathrm{~V}$ or $50 \mathrm{~V}$. At the electrodes absorbing wall boundary conditions for the particles are applied. At boundaries in the $\mathrm{X}$ and $\mathrm{Z}$ directions periodic boundary conditions are used, both for particles and potential. The neutral argon was treated as a fixed background with constant density and temperature, as its density is much higher than the densities of charged species. Only the charged particle dynamics was followed. In the simulation, the plasma was sustained self-consistently due to electron impact ionization of the neutral gas by the electrons accelerated in the applied rf voltage.

Dust particles with radii $\mathrm{R}_{d}=2.5,5.0,7.5$ and $10.0 \mu \mathrm{m}$ were introduced into the discharge with zero starting charge. The position of the dust particles was fixed at three different positions of $\mathrm{y}=0.12,0.49$ and $1.08 \mathrm{~cm}$, which correspond to locations in sheath, presheath and bulk regions respectively (in $\mathrm{x}, \mathrm{z}$ the particles are centered in the simulation domain). The cell in which the dust grain is located together with the neighboring cells form the 
"MD" region, as shown in Fig. 1.

Plasma particles which cross the computational dust grain boundary are assumed to be absorbed. Other charging processes, such as secondary electron emission, or surface photo emission, have not been considered. In our model, the dust potential is derived from the electric field around the dust. From this field, the potential is derived by integration.

\section{RESULTS}

Here, we present the simulation results for dust parameters (dust charge, potential and potential distribution) computed for static spherical dust grains located at the three positions in the rf discharge. The results will also be compared with theoretical models.

\section{A. Background Plasma Discharge Characteristics}

Discharge properties, such as plasma species densities and velocity distributions, govern the charging process of the dust grain. The dust charge itself determines the dust potential and field around the dust, which again influences the shielding and ion drag force. Therefore, the presence of dust will modify the local discharge characteristics. Hence, it is necessary to study the discharge characteristics to gain an insight into the charging processes.

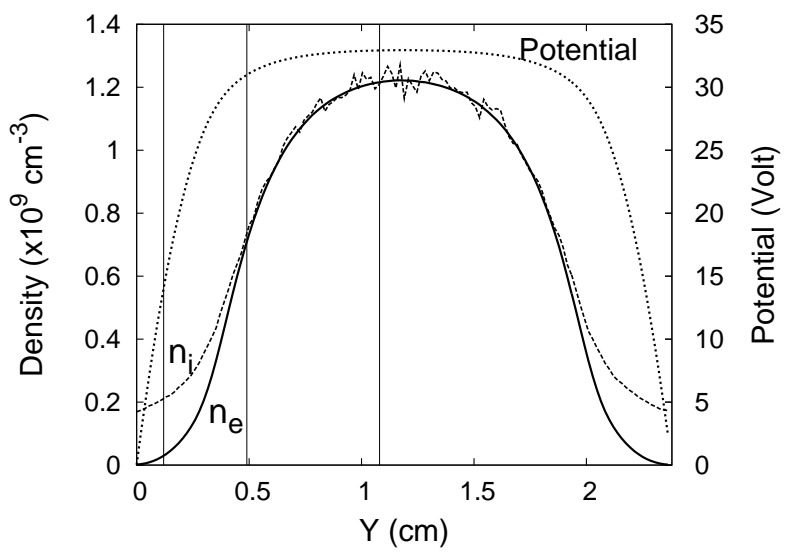

FIG. 2: Time-averaged density and potential profiles. Vertical lines represent the y-coordinates of the dust.

Figure 2 shows the computed electron and ion densities in a discharge between the electrodes on the central axis, for a pressure of $\mathrm{p}=50 \mathrm{~Pa}$. The ion density equals the electron 
density in the bulk satisfying quasi-neutrality and exceeds the electron density in the sheaths. After equilibration, the bulk plasma parameters are $n_{e}=1.22 \times 10^{9} \mathrm{~cm}^{-3}$ and $T_{e}=5.5 \mathrm{eV}$ respectively, yielding an effective electron Debye-length $\lambda_{d}=550 \mu \mathrm{m}$. Similarly, the effective ion Debye-length is $\approx 37 \mu \mathrm{m}\left(T_{i} / T_{e} \cong 0.0045\right)$, which is one order smaller than the electron Debye-length. Fig. 2 also shows the time-averaged potential between electrodes. From the figure, the sheath width can approximately be derived to be about $0.5 \mathrm{~cm}$.

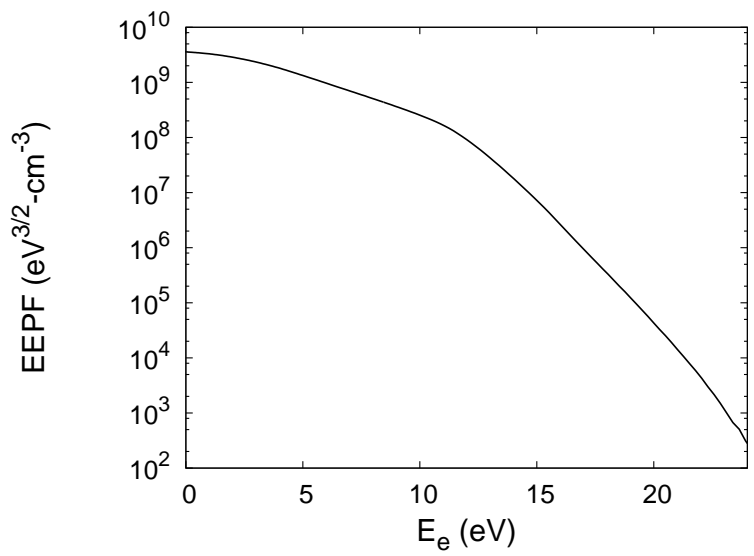

FIG. 3: Time-averaged electron energy probability function

The time-averaged electron energy probability function ${ }^{55}$ at the center of the discharge is shown in Fig. 3. It can be seen that the electron distribution is non-Maxwellian, and rather Druyvesteyn-like ${ }^{22,23,47}$ where a strong drop of high-energy electrons is observed. Such electron distribution in the discharge indicates the Ohmic heating ${ }^{22,47}$ in a Ramsauer gas like argon. Similar electron distributions were experimentally found in low-pressure capacitive rf discharges ${ }^{22}$.

Figure 4 shows the time-averaged ion velocity (normalized to local sound speed) between the electrodes. It can be seen that ions are subsonic even in the sheath, except in a few cells neighboring to electrode. Ions are not accelerated to Bohm Velocity near the plasma-sheath edge. This is due to high collisionality in the system. The mean free path of ion-neutral charge-exchange collisions $\left(\lambda_{m f p}\right)$ is only about 85 micron, which is less than the sheath width. Thus, this is a highly collisional sheath. 


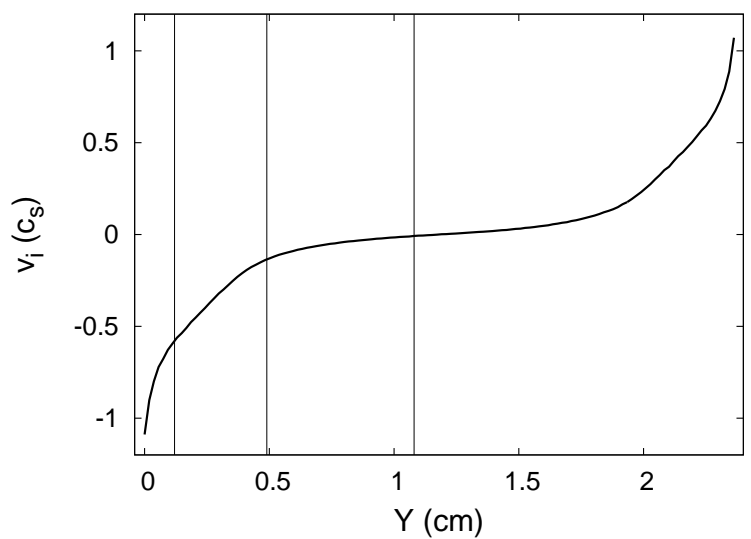

FIG. 4: Time-averaged ion mach number $\left(v_{i} / c_{s}\right)$ along the discharge axis Y. Vertical lines represent the y-coordinates of the dust.

\section{B. Dust potential}

In the so characterized background plasma, we have computed the floating potential on the dust particles of different radii, located at different positions in the rf discharge plasma. During the simulation the dust grains acquired a negative charge by the collection of plasma electrons and ions.

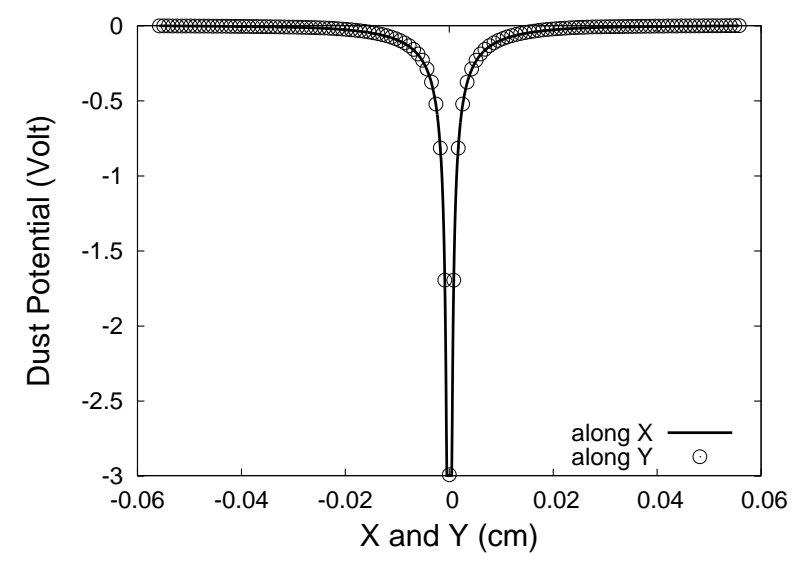

FIG. 5: Spatial dust potential distribution for a 5 micron dust grain located in the plasma bulk

Figure 5 shows the dust potential along the X, Y-axes. It can be seen that the floating potential on the dust grain is about $-2.98 \mathrm{~V}$ and radially symmetric around the dust. In determining the dust potential profile in the sheath, one has to account for the strong change of the plasma potential in the sheath. The potential profile observed in the sheath along $\mathrm{X}$ is symmetric, but is asymmetric around the dust along the Y-direction (which is along 
the electrode separation). This asymmetry is introduced due to the streaming ions in the sheath. The dust potential profile will be analyzed in more detail below.

\section{Dust Potential vs. Dust size \& location}

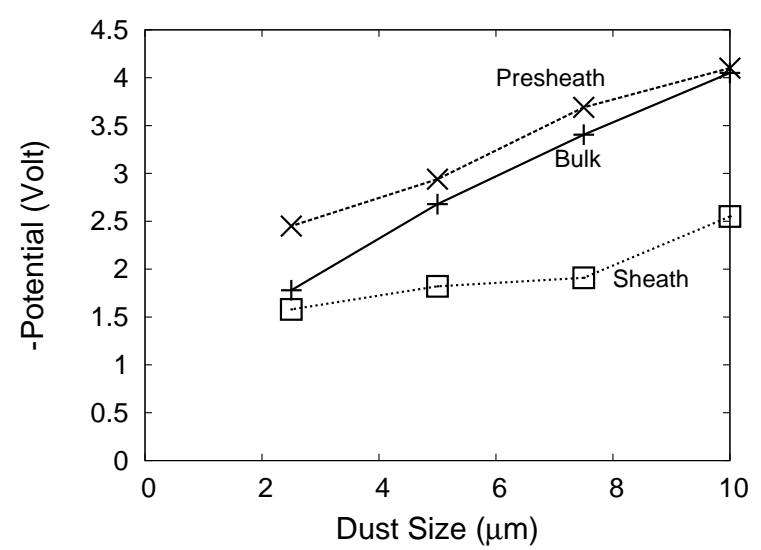

FIG. 6: Dust potential as a function of Dust size

Now the dust potential is analyzed for dust of different sizes (see Fig. 6). It can be seen that the dust potential scales nearly linearly with dust size $\left(\mathrm{R}_{d}\right)$. As already mentioned, the dust potential is computed by the integration of the dust field around it. From the collisionless models like $\mathrm{OML}^{5}$, one expects that the dust potential is independent of dust size. But for this collision-dominated plasma, the ion current $\left(I_{i}\right)$ to the dust grain increases, resulting in a size-dependence other than $\mathrm{R}_{d}^{2}$ in $\mathrm{OML}^{43,44}$. This collisional ion current contribution results in a dust-size dependence of the dust surface potential. These results qualitatively agree with the simulation results of Zobnin et.al. ${ }^{43,44}$, where a dependence of dust potential on dust size has been demonstrated. There, an additional collisional contribution to the ion current onto the dust grain in a stationary plasma has been obtained by solving the kinetic equation accounting for charge-exchange ion-neutral collisions. Zobnin et.al. derived ion current, surface potential, etc., for intermediate collisionality. They showed that the surface potential increases approximately logarithmically ${ }^{43}$ with the dust radius in the hydrodynamic limit $\left(\lambda_{m f p} \ll\right.$ shielding length, $\left.\lambda_{s}\right)$. For particles with $R_{d} / \lambda_{s} \geq T_{i} / T_{e}$ the absolute value of the surface potential increases with the particle size. In our case, for a $5 \mu \mathrm{m}$ particle $R_{d} / \lambda_{s} \approx 0.03, T_{i} / T_{e} \approx 0.0045$ this condition is fulfilled. Zobnin et.al. ${ }^{44}$ also proposed analytical approximations for the ion currents. These analytical formulae show 
that the ion current is a complex function of collisionality and particle size, resulting in a size-dependence of the surface potential. In Fig. 7, dust surface potential for various dust sizes obtained in the present simulations have been compared with the values obtained from the analytical formulae given in [44], for the shielding lengths obtained in our simulations (see below). Both results show the same tendency, but shifted by about 1 volt. This deviation can be attributed to the use of a Maxwellian distribution for electrons in [44], whereas here the distribution is Druyvesteyn-like. The Maxwellian distribution contains more high energy electron population (high energy tail) which contributes additional electron flux to the dust resulting in a more negative dust potential. Hence, dust potential values from Zobnin fit are smaller than the values obtained in our simulations.

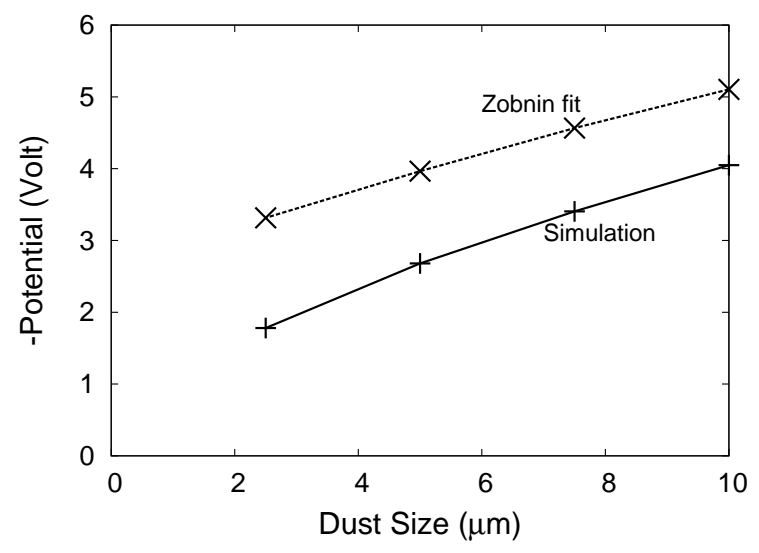

FIG. 7: Dust size dependence of floating potential (in plasma bulk) determined in our simulations, compared to the analytical fit given by Zobin et.al. ${ }^{44}$

\section{Shielding}

We now discuss the dust potential distribution around the dust. The potential around a charge or probe in a plasma is screened or shielded by the plasma particles. It is usually assumed that dust potential screening is a Debye-Hückel (or Yukawa) type. Here, the potential at a distance $\mathrm{R}$ from dust is therefore compared to

$$
\phi_{d}(R)=U_{d} \frac{R_{d}}{R} \exp \left(-\frac{R-R_{d}}{\lambda_{s}}\right)
$$

where $U_{d}$ is the floating potential on the dust. This is an important parameter, which governs the particle interactions. The dust potential around the dust is plotted in units of 
$\log \left(U_{d} R_{d} / R\right)$ versus $\left(X-R_{d}\right)$, as in these units the Yukawa potential is linear. From our kinetic simulations thus the shielding length $\lambda_{s}$ is retrieved.

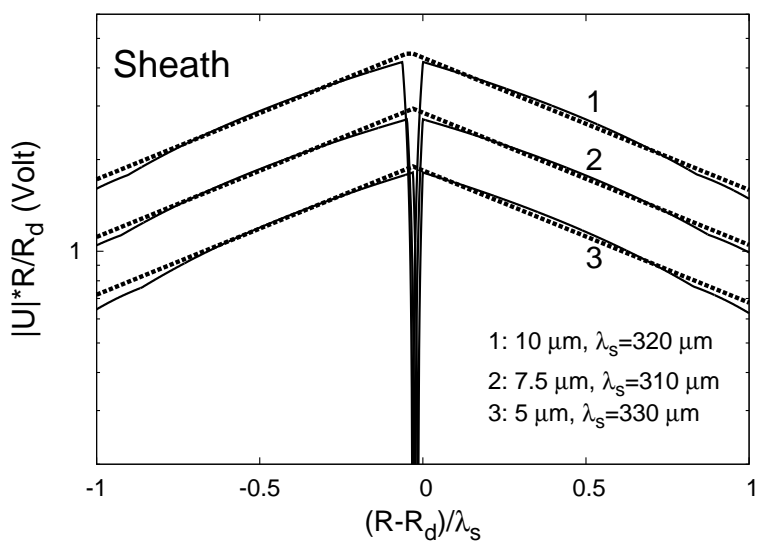

FIG. 8: Computed dust potential in the sheath compared to the Debye-Hückel potential (dashed line) for various sizes of dust. The resulting screening lengths are also listed.

In Fig. 8, the simulated dust potential distributions for various sizes of dust grains located in the sheath are compared to the analytical Debye-Hückel potential. Thus, the dust potential from the $P^{3} M$ code behaves like a Debye-Hückel potential in a region close to the dust particle up to a distance $\mathrm{R}=\lambda_{s}$. Shielding lengths derived for all the cases are about $320 \mu \mathrm{m}$. Moreover, the shielding length is found between the electron and the ion Debye lengths. Hence, it can be concluded that the effective shielding is done both by electrons and ions. Deviations of shielding lengths from ion Debye length have already been discussed in $[15,44,56]$.

In the bulk and the presheath, we find a similar behaviour: the potential is DebyeHückel-like with screening lengths 200 and $220 \mu \mathrm{m}$ respectively. The screening lengths in the presheath and the sheath are larger than the cell size and are reliable. The screening length in the bulk is comparable to the cell size. However, these values are in agreement with the values of the shielding length which are found to fit the charge and potential calculations in Sec. IV F very well. From the bulk to the sheath the ion velocities increase and ion shielding becomes increasingly ineffective. Hence, the shielding length slightly increases from the bulk to the sheath. 


\section{E. Charge evolution on Dust}

We have also computed the charge on dust particles of different radii, located at the three positions in the rf discharge plasma by counting the plasma particles arriving at the numerical particle boundary.

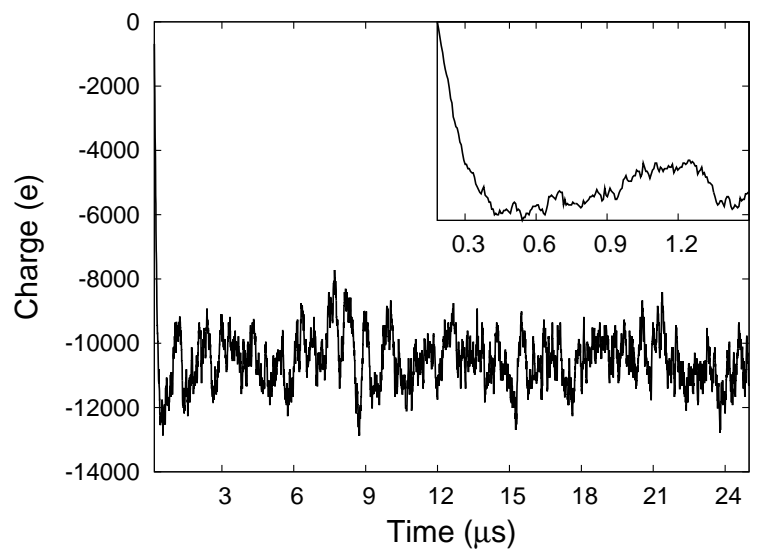

FIG. 9: Temporal evolution of the dust charge for a 5 micron particle in the bulk. The inlet shows a magnification of the first $1.5 \mu$ s of charging.

In Fig. 9, we present the evolution of the electric charge of a dust grain with a radius of $5.0 \mu \mathrm{m}$ located in the plasma bulk. A fast initial charging takes place due to the collection of electrons, while equilibration takes place on the ion time scale (of the order of $0.5 \mu \mathrm{s}$ in our case $)^{45,47}$. The equilibrium dust charge is subject to stochastic fluctuations due to the discrete nature of charge carriers. Stochastic fluctuations have been studied by Cui et. al. ${ }^{57}$, and it has been shown that the amplitude of the stochastic fluctuations is approximately equal to $0.5 \sqrt{Q_{d}}$, where $Q_{d}$ is the equilibrium value of the dust charge. For the $5.0 \mu \mathrm{m}$ dust particle case presented here, $Q_{d}=-10482 \mathrm{e}$ and so the amplitude of the fluctuations should approximately be equal to 51e. In the simulations, the amplitude of the stochastic fluctuations is approximately equal to 825e. This deviation is due to the fact that in the simulation one computational particle represents 89 real electrons or ions.

\section{F. Dust Charge vs. Dust size \& location}

Figure 10 shows the charge evolution for a 5.0 micron dust particle located at the three different positions in the discharge. It can be seen that the charge is reduced in the sheath 


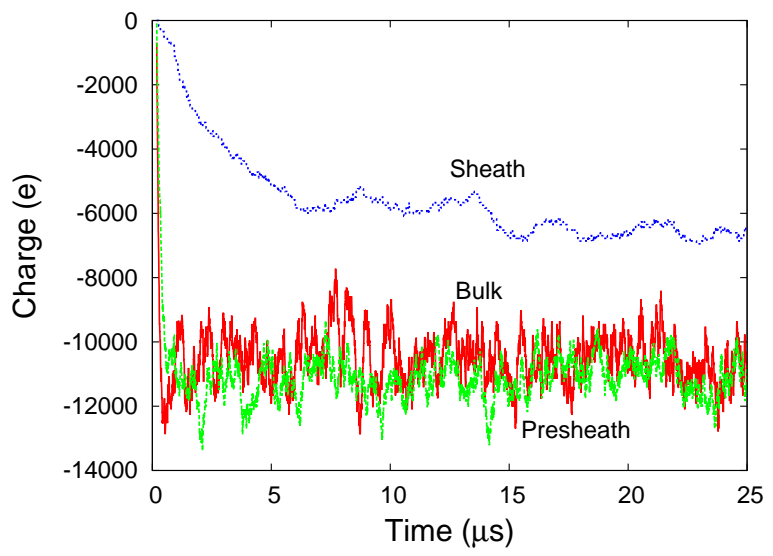

FIG. 10: (Color online) Temporal dust charge evolution of a 5 micron particle for different locations

compared to the bulk or presheath value. The charge in the presheath is equal or slightly larger than the bulk value. This can be explained as follows: Charging is due to electron and ion currents to the dust grain. The electron or ion current is proportional to the density $(n)$, velocity $(v)$ and collection area $\left(\sigma_{d}\right)$ of a dust grain.

$$
I_{e, i} \propto n_{e, i} v_{e, i} \sigma_{d e, i}
$$

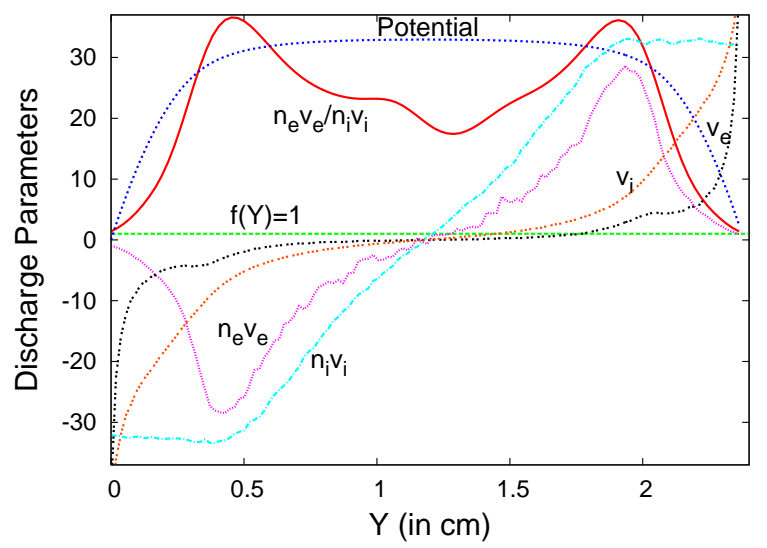

FIG. 11: (Color online) Discharge parameters $\left(v_{e}, v_{i}, n_{e} v_{e}, n_{i} v_{i}\right.$, electron to ion flux ratio, potential) between electrodes. All the parameters are scaled to fit into the scale of flux ratio.

The variation of the dust charge for locations from the bulk to the sheath in a discharge can be explained by analyzing the electron to ion flux-ratio, individual species flux profiles and other discharge parameters along the discharge axis (see Fig. 11). The electron (and ion) 
density decreases from the bulk to the sheath (as shown in Fig. 2), whereas the velocities increase. As the ions, due to high inertia, respond to the average electric field, the ion velocity profile $\left(v_{i}\right)$ resembles the time-averaged potential profile, i.e., the ion velocity monotonically increases from the bulk to the sheath. Electrons respond to the instantaneous electric fields, but due to the strong electric field in the sheath directed towards the electrodes prevents electrons from leaving the plasma for most of the rf cycle. The electrons escape to electrodes during a short time, when the rf sheath collapses. Hence, the electron velocity profile does not follow the average potential drop. The electron flux $\left(n_{e} v_{e}\right)$ increases nonlinearly from bulk to presheath and falls drastically thereafter in the sheath. The ion flux increases almost linearly from bulk to presheath and stays constant beyond the presheath. It is due to the absence of sources and sinks, like recombination, etc. Near the electrodes, the electron and ion fluxes balance each other, as shown by $n_{e} v_{e} / n_{i} v_{i}=1$ in the electron to ion flux-ratio profile. Hence, in case of dust grains located in the presheath and the sheath, ion currents at both locations are almost equal, but the electron current is smaller in the sheath, resulting in a reduced charge in the sheath. The charging time increases when dust is moved from the bulk towards the sheath, due to the same reason. In the presheath, the electron density is comparable to the bulk value, but the effective electron flux has a maximum there. From the flux-ratio profile, $n_{e} v_{e} / n_{i} v_{i}$ value at the presheath is greater than the value at the bulk. Hence, the charge number in presheath is equal to or slightly larger than the bulk value.

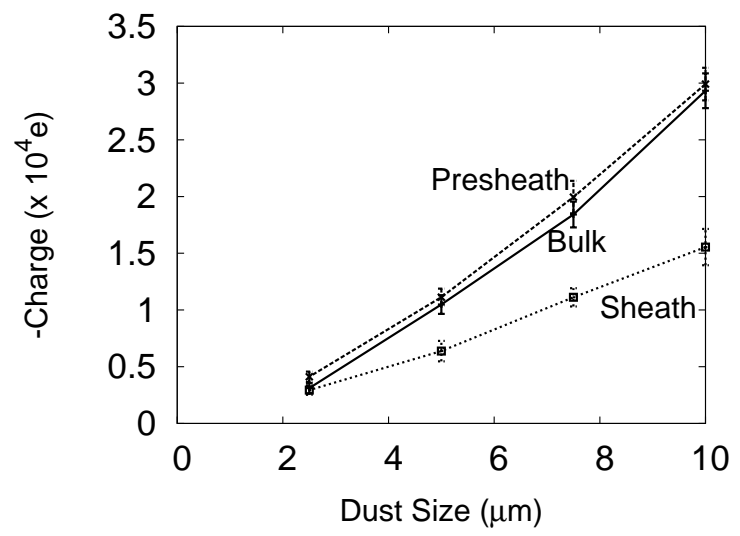

FIG. 12: Dust charge as a function of size for the different locations of the dust grain

Now the dust charge as a function of size for different positions in the plasma is studied (Fig. 12). The charge number on the dust increases nonlinearly with the dust radius. This 
can be understood from the capacitor model, where the charge is given by

$$
Q_{d}=4 \pi \epsilon_{0} r_{d}\left(1+\frac{r_{d}}{\lambda_{s}}\right) \phi_{d}
$$

with the dust potential $\phi_{d}$. As $r_{d} \ll \lambda_{s}$ for the present case, $Q_{d} \propto r_{d} \phi_{d}$. In the previous section, it has been shown that the dust potential $\left(\phi_{d}\right)$ scales almost linearly with the dust size, resulting in a square dependence of charge on dust size.

The dust charge from the simulations is in general reasonable agreement with experiments $^{54,58}$. In experiments on Yukawa balls with particles of $1.7 \mu \mathrm{m}$ radius a charge of about 2000 is found ${ }^{54}$, whereas the simulations yield 2800 charges for a particle of $2.5 \mu \mathrm{m}$. From melting experiments, a charge of about 9000 is measured for a $4.7 \mu \mathrm{m}$ radius particle. Here, the simulations suggest a value of about 10400 for $5.0 \mu \mathrm{m}$ size particle. Shielding lengths can also be derived by fitting above function to the $Q_{d}, \phi_{d}$ data obtained in the simulations. The shielding lengths derived from this method are about $192 \mu \mathrm{m}, 200 \mu \mathrm{m}$, $310 \mu \mathrm{m}$ respectively for dust grains located in bulk, presheath and sheath. These values are consistent with the values obtained from potential distribution curves in Sec. IV D. If the shielding length in the bulk were smaller than the computed value, in the capacitor model the term containing the shielding length would also play a more dominant role and the size-dependence of dust charge would be even stronger than that already obtained.

\section{G. Comparison with Theoretical Models}

Now we discuss the effect of ion-neutral charge-exchange collisions, which are dominant in rf discharges in Argon. In order to do this, simulations have been performed switching-off charge-exchange and excitation collisions. Also, the simulation results are compared with theoretical charging models of $\mathrm{OML}^{5}, \mathrm{ABR}^{6}$, Lampe et. al. ${ }^{15}$ and Khrapak et. al. ${ }^{17}$.

Figure 13 shows the discharge parameters at the three dust locations in the rf discharge obtained in simulations with and without ion-neutral collisions. It can be seen that the effect of collisions is to reduce the net flow velocity. The flow velocity even in the sheath is subsonic, if collisions are considered as discussed above. If collisions are not included, the flow velocity is supersonic, as can be expected by the Bohm criterion.

Figure 14 shows the dust floating potential values obtained without ion-neutral charge exchange collisions and excitation collisions. Also, the dust quantities computed from theo- 


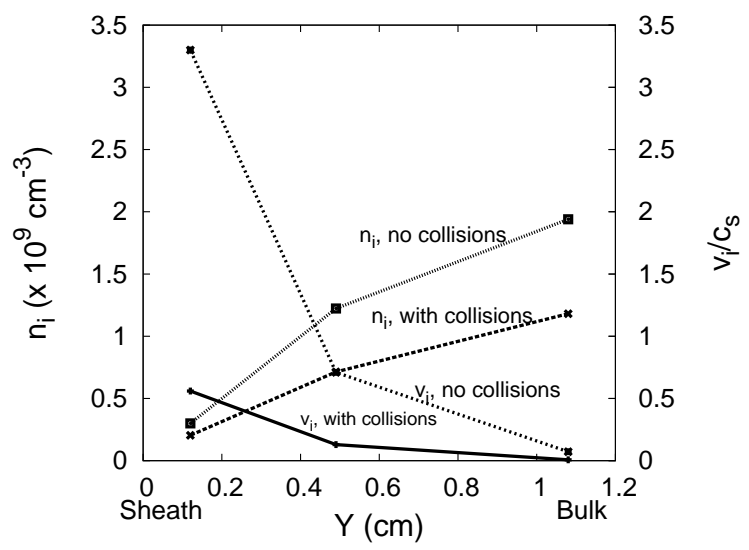

FIG. 13: Comparison of discharge parameters with and without ion-neutral collisions

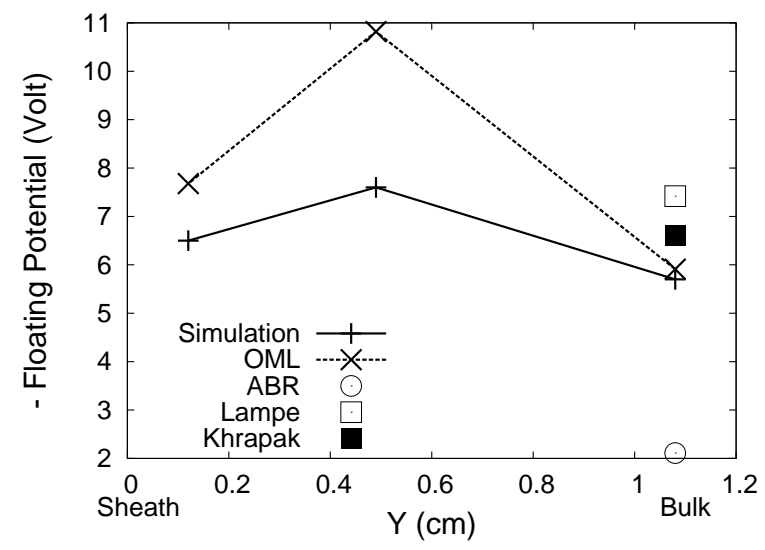

FIG. 14: Comparison of dust floating potential with theoretical models for a $5 \mu \mathrm{m}$ particle in case ion-neutral collisions are not considered

retical models (OML, ABR, Lampe, Khrapak) are presented. ABR model predicts a single value for the dust potential for a given dust size and Debye-length ratio, irrespective of collisionality. Lampe and Khrapak models do not account for streaming conditions and are given for the bulk only here. The dust potential predicted by these models has been used to obtain the dust charge from the capacitance model. In case when collisions are not considered, the dust quantities obtained in simulations are comparable to OML, except in the presheath region. The dust quantities from simulations are larger than ABR and slightly lower than the values obtained from Lampe and Khrapak models. This means that when the collisions are not effective, the ions retain their orbital trajectories and hence the OML is a good approximation.

Figure 15 shows the dust floating potential values obtained with ion-neutral charge ex- 


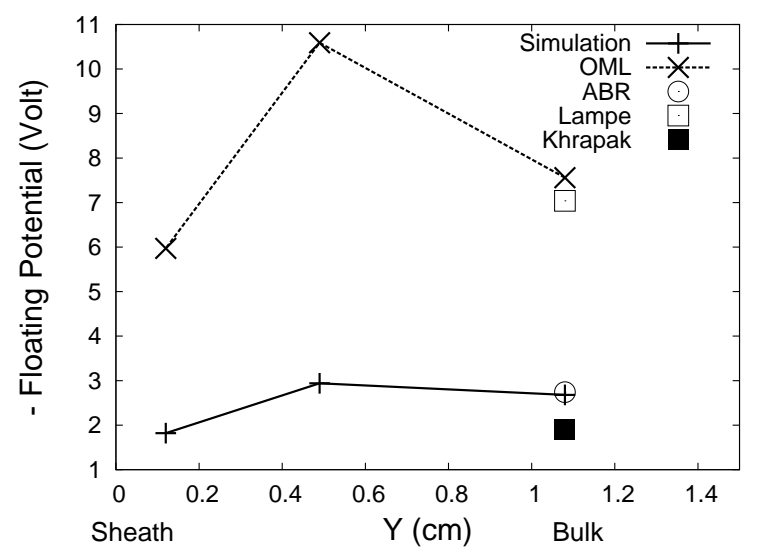

FIG. 15: Comparison of dust floating potential with theoretical models for a $5 \mu \mathrm{m}$ particle in case ion-neutral collisions are considered

change collisions and excitation collisions. In case when collisions are effective, the computed dust quantities are considerably lower than the calculated values from the OML model, but comparable to values obtained from the ABR model. The reason is the following: the role of collisions is to result in lower velocity ions. These low velocity ions get attracted by the dust strongly obtaining radial motion with respect to the dust center. Hence, numerous collisions destroy the orbital motion of the ions. The dust charge obtained from simulations is slightly higher than ABR value, because of non-Maxwellian distribution.

Lampe $^{15}$ and Khrapak ${ }^{17}$ models calculate the dust parameters as a function of collisionality index ${ }^{15}$, which is equal to $\lambda_{s} / \lambda_{m f p}$, where $\lambda_{s}$ is the shielding length and $\lambda_{m f p}$ is the mfp for ion-neutral charge-exchange collisions. For our discharge parameters, $\lambda_{D e} / \lambda_{m f p} \approx 6.5$ and $\lambda_{D i} / \lambda_{m f p} \approx 0.43$. Khrapak ${ }^{17}$ used the ion Debye length as the shielding length. Lampe ${ }^{15}$ used the effective screening length from both the electrons and ions, $\left(\lambda_{D e}^{-2}+\lambda_{D i}^{-2}\right)^{-1 / 2}$ (which is again equal to the ion Debye length for our parameters), as the shielding length. But, our simulation results presented in the previous section show that in the plasma bulk the shielding length is in between electron and ion Debye lengths. As Lampe model predicts the dust quantities only up to a collisionality index 0.5 , we only consider the ion Debye length for calculations. From Fig. 15, it can be seen that the dust quantities obtained from Lampe model are larger than the values obtained in simulations. We compute the dust quantities for Khrapak model, considering only the shielding length obtained in our simulations. Dust parameters obtained from Khrapak model are slightly smaller than the simulation results. 


\section{CONCLUSIONS}

Three-dimensional simulations have been carried out using Particle-Particle-ParticleMesh code, to compute the dust charge and potential on a dust particle located at various positions in an Argon rf discharge. The dust potential distribution has been compared to the Debye-Hückel potential and shielding lengths for dust potential at different locations in rf discharge have been derived. It has been found that in plasma bulk and presheath regions the shielding lengths are in between ion and electron Debye lengths, indicating shielding by both ions and electrons. A linear dependence of dust potential on dust size has been found, which results in nonlinear dependence of the dust charge with the dust size. The computed dust parameters have been compared to OML, ABR, Lampe and Khrapak theoretical models.

\section{Acknowledgments}

This work was supported by SFB-TR24 under Project A4 and Initiative and Networking Fund of the Helmholtz Association. 
1 P. K. Shukla and A. A. Mamun, Introduction to dusty plasma physics, Institute of Physics, Bristol, UK, ISBN 075030653X (2002).

2 A. Melzer, V. A. Schweigert and A. Piel, Physica Scripta, 61, 494 (2000).

3 M. Wolter, A. Melzer, O. Arp, M. Klindworth, M. Kroll and A. Piel, IEEE Trans. on Plasma Science, 35(2), 266 (2007).

4 H. Mott-Smith and I. Langmuir, Physical Review, 28, 727 (1926) .

5 J. E. Allen, Physica Scripta, 45, 497 (1992) .

6 J. E. Allen, R. L. F. Boyd and P. Reynolds, Proc. Phys. Soc. B., 70, 297 (1957) .

7 I. B. Bernstein and I. Rabinowitz, Phys. Fluids, 2, 112 (1959).

8 See National Technical Information Service Document No. AD634596 (J. G. Laframboise, Theory of Spherical and Cylindrical Langmuir Probes in a Collisionless Maxwellian Plasma at Rest, U.T.I.A.S. Report No. 100, 1996). Copies may be ordered from the National Technical Information Serice, Springfield VA 22161.

9 J. E. Allen, B. M. Annaratone and U. de Angelis, J. Plasma Phys., 63, 299 (2000).

10 B. M. Annaratone, M. W. Allen and J. E. Allen, J. Physics D: Applied Phys., 25, 417 (1992).

11 R. V. Kennedy and J. E. Allen, J. Plasma Physics, 67, 243 (2002).

12 R. V. Kennedy and J. E. Allen, J. Plasma Physics, 69, 485 (2003).

13 D. Bohm, The Characteristics of Electrical Discharges in Magnetic Fields, ed. A. Guthrie and R.K. Wakerling (New York: McGraw-Hill 1949).

14 M. Lampe, V. Gavrishchaka, G. Ganguli and G. Joyce, Phys. Rev. Lett., 86(23), 5278 (2001).

15 M. Lampe, R. Goswami, Z. Sternovsky, S. Robertson, V. Gavrishchaka, G. Ganguli and G. Joyce, Physics of Plasmas, 10(5), 1500 (2003).

16 S. V. Ratynskaia, S. A. Khrapak, A. V. Zobnin, M. H. Thoma, M. Kretschmer, A. D. Usachev, V. V. Yaroshenko, R. A. Quinn, G. E. Morfill, O. F. Petrov and V. E. Fortov, Phys. Rev. Lett., 93(8), 085001 (2004).

17 S. A. Khrapak, S. V. Ratynskaia, A. V. Zobnin, A. D. Usachev, V. V. Yaroshenko, M. H. Thoma, M. Kretschmer, H. Höfner, G. E. Morfill, O. F. Petrov and V. E. Fortov, Phys. Rev., $72,016406(2005)$.

18 Y. L. Alpert and A. V. Gurevich and L. P. Pitaevskii, Space Physics with Artificial Satellites 
(New York: Plenum Press, 1965).

19 F.F. Chen, J. Nucl. Energy C: Plasma Phys., 7, 47 (1965).

20 R. N. Nowlin and R.N. Carlile, J. Vacuum Sci. Technol. A, 9(5), 2825 (1991).

21 J. E. Daugherty, R. K. Porteous, M. D. Kilgore and D. B. Graves, J. Appl. Phys., 72(9), 3934 (1992).

22 V.A. Godyak and R.B.Piejak, Phys. Rev. Lett., 65, 996 (1990).

23 K. Matyash and R. Schneider, Contributions to Plasma Physics, 44(7-8), 589 (2004).

24 A. Melzer, T. Trottenberg and A. Piel, Phys. Lett. A, 191, 301 (1994).

25 T. Trottenberg, A. Melzer and A. Piel, Plasma Sources Sciences and Technology, 4, 450 (1995).

26 U. Kortshagen and G. Mümken, Physics Letters, A, 217, 126 (1996).

27 A. Homann, A. Melzer and A. Piel, Physical Review E, 59(4), R3835 (1999).

28 E. B. Tomme, B. M. Annaratone and J E Allen, Plasma Sources Sci. Technol., 9, 87 (2000).

29 E. B. Tomme, D. A. Law, B. M. Annaratone and J E Allen, Phys. Rev. Lett., 85(12), 2518 (2000).

30 U. Konopka, L. Ratke and H. M. Thomas, Phys. Rev. Lett., 79(7), 1269 (1997).

31 U. Konopka, G. E. Morfill and L. Ratke, Phys. Rev. Lett., 84(5), 891 (2000).

32 G. E. Morfill, H. M. Thomas, U. Konopka and M. Zuzic, Physics of Plasmas, 6(5), 1769 (1999).

33 S. Nunomura, T. Misawa, N. Ohno and S. Takamura, Phys. Rev. Lett., 83(10), 1970 (1999).

34 B. Liu, K. Avinash and J. Goree, Phys. Rev. E, 69, 036410 (2004).

35 A. Homann, A. Melzer, S. Peters and A. Piel, Physical Review E, 56, 7138 (1997).

36 B. Walch, M. Horanyi and S. Robertson, IEEE Trans. Plasma Sci., 22(2), 97 (1994).

37 Z. Sternovsky, M. Horanyi and S. Robertson, J. Vacuum Sci. Technol. A, 19, 2533 (2001).

38 I.H.Hutchinson, Plasma Phys. Control. Fusion, 44, 1953 (2002).

39 I.H.Hutchinson, Plasma Phys. Control. Fusion, 45, 1477 (2003).

40 I.H.Hutchinson, Plasma Phys. Control. Fusion, 47, 71 (2005).

41 I.H.Hutchinson, Plasma Phys. Control. Fusion, 48, 185 (2006).

42 I.H.Hutchinson and L.Patacchini, Phys. of Plasmas, 14, 013505 (2007).

43 A. V. Zobnin, A. P. Nefedov, V. A. Sinel'shchikov and V. E. Fortov, JETP, 91 (3), 483-487 (2000).

44 A. V. Zobnin, A. D. Usachev, O. F. Petrov and V. E. Fortov, Phys. Plasmas, 15, 043705 (2008).

45 K. Matyash and R. Schneider, J. Plasma Phys., 72(6), 809 (2006). 
46 K. Matyash, R. Schneider, F. Taccogna and D. Tskhakaya, J. Nuclear Materials, 363-365, 458 (2007).

47 K. Matyash, R. Schneider, F. Taccogna, A. Hatayama, S. Longo, M. Capitelli, D. Tskhakaya and F. X. Bronold, Contrib. Plasma Phys., 47(8-9), 595 (2007).

48 C. K. Birdsall and A. B. Langdon, Plasma physics via computer simulation (McGraw-Hill, New York, 1985).

49 R. Smirnov, Y. Tomita, T. Takizuka, A. Takayama and Yu. Chutov, Contrib. Plasma Phys., 44(1-3), 150 (2004).

50 R. Smirnov, Y. Tomita, D. Tskhakaya and T. Takizuka, Contrib. Plasma Phys., 46(7-9), 623 (2006).

51 V. Vahedi and M. Surendra, Computer Physics Communications, 87(1-2), 179 (1995).

52 T. Takizuka and H. Abe, J. Comp. Phys., 25, 205 (1977).

53 A. V. Phelps, (unpublished) ftp://jila.colorado.edu/collision_data.

54 O. Arp, D. Block, M. Klindworth and A. Piel, Phys. Plasmas, 12, 122102 (2005).

55 M.A. Lieberman and A.J. Lichtenberg, Principles of Plasma Discharges and Materials Processing, (New York: Wiley, 1994).

56 M. Lampe, G. Joyce and G. Ganguli, Phys. Plasmas, 7(10), 3851 (2000).

57 C. Cui and J. Goree, IEEE Trans. on Plasma Science, 22, 151 (1994).

58 A. Melzer, A. Homann and A. Piel, Phys. Rev. E, 53, 2757 (1996). 